\title{
Escuela tomada. El movimiento estudiantil secundario rosarino en el gobierno camporista
}

\author{
Occupied School. The high school student movement in Rosario in the Cámpora \\ government
}

\author{
Laura Luciani \\ lauluciani@gmail.com \\ Centro Latinoamericano de Investigaciones en \\ Historia Oral y Social. Universidad Nacional de \\ Rosario (CLIHOS-UNR), Argentina \\ Investigaciones Socio-históricas Regionales (ISHIR). \\ CONICET, Argentina
}

Recepción: 17 Agosto 2020

Aprobación: 18 Noviembre 2020

Publicación: 01 Marzo 2022

Cita sugerida: Luciani, L. (2022). Escuela tomada. El movimiento estudiantil secundario rosarino en el gobierno camporista. Sociohistórica, 49, e158. https://doi.org/10.24215/18521606e158

\begin{abstract}
Resumen: El artículo indaga en las ocupaciones de escuelas secundarias producidas entre mayo y julio de 1973, centrando el análisis en la ciudad de Rosario. El estudio, desarrollado a partir del trabajo con fuentes documentales y entrevistas, permite advertir la significación del movimiento estudiantil secundario, sus dinámicas de acción, demandas y experiencias en un contexto de fuerte radicalización política en el inicio del gobierno de Héctor Cámpora.

La hipótesis de este artículo es que las tomas constituyeron un acontecimiento central en la reconfiguración del movimiento secundario que en general ha sido minusvalorado. Al mismo tiempo sostiene que, más allá de la retórica contra el continuismo, las tomas habilitaron reivindicaciones específicas que marcaron la agenda del movimiento. Por último, se enfatiza en un diverso abanico de agrupaciones políticas que participaron de la agitación en las instituciones educativas, en el que se destacan por su crecimiento y proyección en los meses siguientes las agrupaciones peronistas.
\end{abstract}

Palabras clave: Estudiantes, Ocupación, Militancia política, Rosario.

Abstract: The article examines the high schools' occupations between May and July 1973, focusing the analysis on the city of Rosario. The study, developed from research with documentary sources and interviews, allows us to understand the significance of the high school student movement, its dynamics of action, demands and experiences in a context of strong political radicalization in the beginning of the government of Héctor Cámpora.

The hypothesis of this article is that the occupied of schools were a central event in the reconfiguration of the high school student's movement, which has been generally underestimated. At the same time, it is argued that beyond the rhetoric against continuismo, the occupation enabled specific demands that set the agenda for the movement. Finally, a diverse range of political groups participated in the occupation of the educational institutions where the Peronist groups stood out for their growth and projection in the following months.

Keywords: Students, Occupation, Politics militancy, Rosario. 


\section{INTRODUCCIÓN}

Los estudios sobre movimientos estudiantiles y participación política de estudiantes secundarios en Argentina han tenido hasta el momento escasa relevancia y son pocos los que han indagado en sus manifestaciones públicas, demandas, escenarios de acción. ${ }^{1}$ Asimismo, las investigaciones desarrolladas señalan como característica la intermitencia con la cual emergieron en coyunturas específicas, así como su asociación con el movimiento universitario, aspecto que en ocasiones tiende a solapar su actuación y convertirla en subsidiaria de un colectivo más general (Larrondo, 2013, p. 8 y ss.).

Esa lectura colabora en la invisibilización de procesos en los que la acción de estudiantes secundarios fue central. En este artículo nos proponemos indagar en una coyuntura que admite un análisis pormenorizado respecto de las dinámicas que asumieron las ocupaciones de escuelas producidas durante el otoño de 1973. Se trató de un momento de fuerte efervescencia y participación que, como sostenemos en este trabajo, les permitió gestar una agenda autónoma del movimiento universitario y convertirse en un punto de inflexión en la definición de sus propias demandas.

Abordaremos las experiencias en la ciudad de Rosario no sólo porque consideramos que acotar la lente a un espacio urbano permite entenderlas en toda su complejidad sino también porque allí las tomas -término con el cual se han popularizado las ocupaciones- se expandieron por una diversidad de escuelas y alcanzaron a casi su totalidad, tema sobre el cual volveremos más adelante. Para ello, iniciamos con una breve introducción a los procesos de rebelión de secundarios previos al año 1973, para luego centrarnos en Rosario hacia finales de la dictadura, específicamente durante la presidencia de facto del general Alejandro Lanusse y los inicios del gobierno de Héctor J. Cámpora. Por último, analizamos algunas tomas de escuelas a través de fuentes documentales y de las voces de sus participantes. ${ }^{2}$ Esa cartografía permite comprender la articulación entre el proceso de agitación social que se impuso en los establecimientos por un breve período y la emergencia y/o crecimiento de agrupaciones políticas en ellos. Asimismo, ese análisis permite identificar algunos rasgos específicos de la militancia secundaria, lo cual evidencia que, lejos de ser dirigidas desde arriba y entendidas exclusivamente como un proceso de apoyo al gobierno de Cámpora y contra el continuismo de la dictadura, las ocupaciones gestaron un activismo complejo, engarzado desde las particularidades de las instituciones, de las demandas estudiantiles que se articularon con ese proceso nacional más general, y a la vez lo tensaron.

\section{EL MOVIMIENTO ESTUDIANTIL SECUNDARIO: UN BREVE ACERCAMIENTO}

La acción política de estudiantes de escuelas en la segunda mitad del siglo XX convoca a reseñar algunas problemáticas referentes a sus condiciones de emergencia y sus posteriores recorridos. En primer lugar, cabe destacar que la extensión de la matrícula se duplicó entre 1945 y 1955. Según Más Rocha (2016, p. 77), en ese lapso creció de 167.116 a 377.713 alumnos y lo hizo principalmente en la terminalidad industrial, seguida de la comercial y la normal, con una mayor concentración en grandes ciudades, principalmente en Buenos Aires (Cammarota, 2011). De igual modo, se evidencia que hacia la segunda mitad de siglo se produjo el aumento de la matriculación de mujeres en la educación media, que osciló de $47 \%$ a $50 \%$ entre la década de 1950 y la siguiente (Manzano, 2011, p. 43).

El rasgo más significativo fue la alta politización que se produjo al calor de algunos conflictos específicos. Aun cuando la agremiación secundaria había sido limitada ya desde 1936 mediante la denominada resolución de la Torre (Más Rocha, 2016) y refrendada posteriormente mediante el decreto 150073 de 1943 (art. 172 y ss.), es posible pensar que, al calor de este crecimiento poblacional y frente a medidas políticas controvertidas, el movimiento estudiantil se congregó mediante federaciones y ligas en un escenario hegemonizado por algunas agrupaciones de izquierda ya existentes. Un primer escenario lo constituyeron las manifestaciones en torno al debate conocido como "Laica o Libre" durante el gobierno de Frondizi, cuando estudiantes 
de escuelas públicas acompañaron mayoritariamente la lucha a favor de la educación laica y, en menor proporción, a favor de la libre. Diversas investigaciones (Manzano, 2009; Larrondo, 2013) coinciden en que este fue un momento de significativa acción de los secundarios a nivel nacional. Para Manzano, la participación por la laica no estuvo condicionada al acompañamiento de la movilización universitaria, sino que asumió cierta especificidad (como la lucha por la derogación de la resolución de la Torre) que se articuló con el conflicto general. Asimismo, en algunos espacios regionales precisamente fueron los estudiantes de escuelas públicas los que iniciaron las acciones públicas. Micheletti (2018, p. 68 y ss.) señala que, en Rosario, las jornadas que comenzaron en mayo de 1958 estuvieron propiciadas por la FRES (Federación Rosarina de Estudiantes Secundarios), que coordinó las tomas de los establecimientos Normal 1, 2 y 3 y el Nacional 1, ${ }^{3}$ a las cuales luego se plegaron las acciones en otros colegios y, posteriormente, estudiantes universitarios que ocuparon las Facultades. En línea similar, Bartolucci (2008) destaca que en Mar del Plata, donde no existían institutos de educación superior, las escuelas fueron el escenario central para el desarrollo del conflicto y advierte sobre la intensa polarización que se tramó entre su alumnado.

Otra coyuntura de fuerte activación se produjo hacia 1968 y 1969. En el caso de Rosario, la agitación desarrollada desde las aulas les permitió a los estudiantes secundarios incorporarse a la protesta social conocida como los Rosariazos tanto en mayo como en septiembre de 1969. No olvidemos que en 1968 el gobierno de Onganía se propuso una reforma educativa amplia (De Luca, 2006), que incluía una modificación del sistema de formación docente con el objetivo de quitar centralidad a las escuelas normales y potenciar la gestación de un nivel superior. Esta reforma generó un alto rechazo en el sector docente (Gudelevicius, 2011; Rodríguez, 2019), que reactivó la movilización de estudiantes de escuelas normales. En Rosario, ese proceso habilitó la rearticulación de la instancia coordinadora mediante la FRES, que se posicionó contra la política del onganiato. Por ello, al momento de las álgidas movilizaciones de 1969, los y las estudiantes secundarios asumieron también una participación activa:

En el 68 funcionábamos en el local de la CGT, en la calle Córdoba. La FRES funcionaba ahí y bueno, se intenta hacer unas cosas (...) cuando viene el 69, el Rosariazo, la FRES convoca; entre el asesinato de Bello y la marcha del silencio, se convoca a un acto secundario en la Melipal [La galería donde matan al estudiante Bello] a llevar flores (...) una cosa de ese tipo y éramos como cien secundarios, una cosa absolutamente llamativa. Bueno, el 21 paramos. ${ }^{4}$

Al igual que en mayo, en septiembre se incorporaron a la acción callejera, dando cuenta de una articulación ya no sólo con universitarios sino también con el movimiento obrero que lideró la protesta social. En Córdoba el movimiento secundario también acompañó la manifestación y la FES (Federación de Estudiantes Secundarios) planificó acciones conjuntas para las actividades desplegadas en ese mes (Ferrero, 2009, p. 193). Asimismo, Bonavena (2013) destaca el accionar a partir del Cordobazo tanto en esa ciudad como en Tucumán, Salta, Mendoza, Bahía Blanca, entre 1969 y 1971. Hacia finales de la dictadura de Lanusse y apenas unos meses antes del inicio de las tomas, en las escuelas técnicas, especialmente de la ciudad de Buenos Aires, se abrió un ciclo de protestas contra la llamada ley Fantasma, una iniciativa provincial que limitaba los alcances de los diplomas otorgados por el Consejo Nacional de Escuelas Técnicas (Bonavena y Millán, 2013). En ese contexto, comenzaron a tener fuerte incidencia viejas y nuevas agrupaciones que, al igual que en el resto del país, iniciaron un camino de reivindicaciones específicas.

Lo expuesto permite ponderar que para 1973 la acción estudiantil tenía sólidos mojones sobre los cuales asentarse, momentos de acumulación en las estrategias de lucha y organización que se recuperaron en un nuevo escenario. No obstante, la agitación social que se extendió en 1973 tuvo un cariz diferente y la movilización adquirió un carácter masivo, con un conjunto de demandas diferenciadas que permitió a la acción de estudiantes secundarios gestar cierta dinámica autónoma, al menos entre 1973 y 1975, cuando se articuló en un contexto de radicalización política más general. En el apartado siguiente indagaremos en este peculiar proceso. 


\section{LA POLITIZACión ESTUdiantil EN EL gOBIERNo DE CÁMPORA}

El inicio de una nueva década señalaba horizontes convulsos y de fuerte radicalidad en Argentina. Luego de los llamados "azos" de 1969 quedaba en evidencia que el proyecto político de los militares instalado con la dictadura en 1966 estaba en crisis y convocaba nuevos acuerdos que incluyeran a aquel actor largamente proscripto: el peronismo. Se sumaba en ese escenario un proceso de radicalización que quedaba explícitamente expuesto con la emergencia y crecimiento de las organizaciones político-militares pero, al mismo tiempo, las desbordaba y señalaba el trastrocamiento profundo en diversos ámbitos de la sociedad (Lenci, 1999). Agustín Lanusse, el último de los dictadores de la autoproclamada Revolución Argentina, asumió el Gran Acuerdo Nacional como una salida electoral que incluyó al peronismo en la contienda. Para 1972, y ante el fracaso de ese acuerdo, se planteaba un escenario que tensionaba y articulaba discursos y prácticas de transformación revolucionaria con la salida electoral, democrática, y trasvasaba distintos espacios. De allí que los posicionamientos respecto de las elecciones abrieron un conjunto de debates intensos en la militancia política, dentro de los viejos y nuevos partidos, organizaciones armadas, organizaciones de base nacientes, ámbitos sindicales y estudiantiles, con lo que se ampliaba el sentido de la participación política y la democratización (Luciani, 2018).

Los y las jóvenes se sumaron a ese proceso en claves diferentes. En primer lugar, acompañaron las candidaturas y la agitación frente a las elecciones. Las organizaciones peronistas que apoyaron la candidatura de Cámpora - Solano Lima y el retorno de Perón lo hicieron a través de la campaña conocida como "Luche y Vuelve". La participación en ese escenario implicó variantes y matices en posicionamientos respecto de la caracterización de la dictadura, la salida electoral y el regreso del viejo líder a escena. En esa complejidad, gran parte de los y las jóvenes se acercaron a la militancia mediante el reparto de volantes y panfletos, con la lectura y venta de las publicaciones militantes, pero también con la presencia en los actos y las movilizaciones que acompañaron el primer retorno de Perón en noviembre de 1972 y el más trágico de junio de 1973. Pero no sólo jóvenes peronistas activaron entonces. Claudia, militante de la Federación Juvenil Comunista (FJC), recuerda que promocionaba la fórmula de Alende - Sueldo y "se militaron todo el verano, yo recuerdo pasar carnavales caminando Provincias Unidas".

En parte, ello se tradujo en las elecciones, que convocaron una participación singularmente alta: doce millones de personas votaron y se incorporó un $25 \%$ de la población que lo hacía por primera vez, es decir votantes entre 18 y 28 años. El 25 de mayo de 1973 se visibilizó con mayor intensidad esa movilización juvenil cuando prorrumpieron en las calles con el objetivo de acompañar la asunción del presidente, el repudio generalizado a los militares salientes y a los miembros de la Junta Militar que escaparon en helicóptero o para reclamar frente a la cárcel de Devoto por la liberación de presos políticos (Sanguinetti, 2013), hecho en el que perdieron la vida dos estudiantes secundarios: uno de Vanguardia Comunista, otro militante de la UES. ${ }^{6}$

La participación masiva de jóvenes también se verificó en la ocupación de instituciones educativas que se produjo entre mayo y julio de 1973 . $^{7}$ Según las investigaciones existentes (Nievas, 1999; Nievas, 2000), comprendieron fundamentalmente a instituciones públicas, y en menor medida al ámbito privado. Fueron portada en la prensa nacional pero fundamentalmente en las revistas militantes que relevaban los acontecimientos en las diversas regiones del país. Si bien tiende a leerse que ese proceso tuvo como objetivo cuestionar el "continuismo" de funcionarios de la dictadura y acompañar la nueva gestión de gobierno, lo cierto es que su desarrollo fue complejo, se radicalizó en poco tiempo y evidenció las tensiones entre el gobierno recién asumido y las demandas emergentes en cada caso (Svampa, 2003, p. 401). Ello se advierte en los fundamentos de un pedido de legisladores sobre la situación, en el que expresaban que los hechos ocurridos atentaban contra la institucionalidad y estaban por fuera del "programa de gobierno de ninguna fuerza política" ${ }^{8}$ Asimismo, el ministro de Educación, Jorge Alberto Taiana, señaló el rechazo a "los vejámenes y 
la violencia física contra las personas y los establecimientos", en referencia a lo ocurrido en los espacios de competencia de su cartera.?

Si nos remitimos específicamente a ese ámbito, fueron afectados edificios de las universidades públicas y privadas, institutos de educación superior no universitaria (profesorados) y escuelas secundarias diversas: públicas y privadas; nacionales y provinciales; normales, comerciales y técnicas, en grandes y pequeñas ciudades del país; es decir, excedió a las grandes urbes y sus establecimientos. Esta particularidad permite asumir que el proceso de radicalización se amplió significativamente entre estudiantes secundarios, incluso en ciudades donde la movilización estudiantil prácticamente no había existido en otras coyunturas. ${ }^{10}$ Siguiendo el ritmo general, el pico se registró hacia la primera quincena de junio, y se revirtió en las semanas siguientes y de cara a la llegada de Perón al país, aunque la conflictividad se mantuvo en situaciones particulares hasta luego del receso invernal, y en algunas instituciones asumieron una dinámica específica.

Como ya hemos señalado, en Rosario la toma de establecimientos educativos fue gravitante, al punto que se sostiene que excepto dos el resto de las escuelas fueron ocupadas (Nievas, 1999, p. 355). Las tomas constituyeron más de un tercio de todas las acciones producidas en la ciudad, índice que es además uno de los más altos del país, pues supera en porcentaje a ciudades como Buenos Aires y Córdoba (Nievas, 2000, p. 165). Según relevamientos propios, en Rosario alcanzó a casi la totalidad de los establecimientos, incluyendo las escuelas nacionales, normales y técnicas; también se registraron algunas escuelas provinciales. En las escuelas confesionales, el Arzobispado a cargo de Monseñor Bolatti decidió levantar las clases en "salvaguardo de la integridad de las personas y bienes" aunque ello no impidió la acción estudiantil en algunas de las instituciones bajo su órbita. ${ }^{11}$

Algunos matices deben ser señalados. En primer lugar, en establecimientos que compartían edificio con institutos de educación superior no universitaria la agitación fue el resultado de estudiantes de este nivel sin participación de secundarios (este sería el caso del Normal 1, por ejemplo). Asimismo, no significó el acompañamiento de todo el alumnado, que en general se mantuvo más bien al margen o menos involucrado en ese proceso. Por ello, realizar un análisis exclusivamente desde la ocupación del edificio no permite ponderar diversas variables que concurren a la hora de reflexionar sobre los alcances del suceso De todos modos, Rosario fue epicentro de las tomas de escuelas.

Los motivos por los cuales este acontecimiento configuró cierta centralidad en la región deben ser indagados. Uno de los primeros a considerar es la politización en el ámbito escolar que, como hemos señalado en el apartado anterior, tenía una larga tradición en la ciudad y que se resignificó en los meses previos, especialmente en algunas escuelas y frente a determinados problemas específicos. En 1972, al igual que en otras ciudades del país, los estudiantes de escuelas técnicas de la provincia se organizaron en pos de plantear un reconocimiento al título técnico y contra CONET, ya que consideraban que no representaba sus intereses. Asimismo, hacia finales de año se propuso la creación de una coordinadora nacional a raíz del encuentro de estudiantes técnicos de diversas partes del país en La Rioja, en el que los jóvenes santafesinos participaron. ${ }^{12}$ En este sentido, es posible advertir que el proceso de activación en establecimientos de enseñanza técnica durante los últimos meses de 1972 incidió significativamente en la politización de escuelas técnicas de la ciudad y en la organización de sus estudiantes, al punto que para junio de 1973 todas las instituciones dependientes de $\mathrm{CONET}^{13}$ y algunas escuelas técnicas provinciales fueron ocupadas.

Otros de los factores a considerar son de corte regional y local. El primero, los comicios en la provincia. En Santa Fe, la campaña electoral se extendió unos días más, hasta el 15 de abril, con la definición en segunda vuelta de la fórmula Silvestre Begnis - Eduardo Cuello para la gobernación. La postergación significó nuevos actos políticos, en los que se involucró la mayoría de las agrupaciones juveniles, fundamental pero no únicamente aquellas pertenecientes al arco peronista. El 11 de abril, el mismo día que Cámpora respaldaba en la capital provincial la candidatura oficialista, la Juventud Peronista, Regional II, realizó un mitin en Rosario que contó con la asistencia de 1600 personas. ${ }^{14} \mathrm{Al}$ día siguiente la mesa de Juventud del FREJULI 
realizó otro en plaza Pringles, con menor concurrencia. ${ }^{15}$ Para la misma fecha, Jorge Obeid, referente juvenil en la provincia, señalaba: "En este momento la juventud peronista se encuentra abocada al lanzamiento de una propuesta a nivel nacional de movilización nacional de toda la juventud argentina (peronistas y no peronistas), en pos de la garantización (sic) de la entrega del gobierno". ${ }^{16}$ En este sentido, es posible asumir que el alargamiento de la contienda electoral supuso la continuidad de la participación activa en la calles de diversas agrupaciones políticas que involucraban a jóvenes.

Por último, reseñamos un hecho represivo que también impactó sobre el alumnado: el secuestro y desaparición del estudiante de Ciencias Económicas y militante de la UEL Ángel "Tacuarita" Brandazza ocurrido en noviembre de 1972 (Águila, 2017). Ese suceso, las acciones públicas de sus familiares ante su desaparición y la creación de la comisión Bicameral de la provincia que se dedicó a investigar el hecho (Scocco, 2018) son rememorados por las personas entrevistadas como acontecimientos clave en los inicios de su militancia estudiantil. Estos aspectos nos permiten dimensionar que la agitación política de estudiantes secundarios mantuvo en la ciudad algunas claves comunes a la politización más general, pero se combinaron con situaciones específicas y permitieron que hacia junio de 1973 los y las estudiantes se encontrasen en un tiempo marcado por la intensa movilización.

\section{LAS TOMAS, UN ACERCAMIENTO DESDE Rosario17}

En los recuerdos, los días que transcurrieron entre finales de 1972 y la masacre de Ezeiza fueron de gran convulsión, de acciones, movilizaciones, protesta, organización, ocupación, movilización en la calle, creación de coordinadoras, entre muchas otras actividades. Para quienes participaron de esos acontecimientos, el tiempo es un pliegue rugoso, imposible de hilvanar en una cadencia progresiva que diferencie los primeros intentos de organización hasta la intervención en las escuelas o las movilizaciones posteriores. Las tomas se convirtieron en una fuerza centrípeta que concentró sobre sí misma procesos concomitantes y complejos; motivaciones, intereses, prácticas políticas, identidades en construcción. Es un tiempo suspendido, que para algunas personas fue efímero y para otras, extenso.

No obstante, las narrativas parten de construir una historia que se habilita con el llamado de apertura política, momento en el cual se inició un lento y clandestino trabajo para organizar el centro de estudiantes que convocó a viejas y nuevas tradiciones políticas en el seno de las escuelas. A la Federación Juvenil Comunista, la más tradicional de las agrupaciones estudiantiles, y a las vinculadas al Partido Socialista se sumaban trotskistas como la TERS (Tendencia Estudiantil Revolucionaria Socialista) y la Juventud Socialista de Avanzada, así como diversas agrupaciones peronistas que, a medida que avanzó el proceso de radicalización, fueron definiendo sus posiciones. Entre 1972 y las tomas, dos organizaciones emergieron de ese espacio político: la JSP (Juventud de Secundarios Peronistas) vinculada al FEN (Frente Estudiantil Nacional), por un lado, y la UES (Unión de Estudiantes Secundarios), por otro. Esta última se reconstituyó en la provincia a partir del mes de abril y en ella confluyeron dos experiencias previas: el Movimiento de Acción Secundaria, más cercano a las Fuerzas Armadas Revolucionarias (FAR), y Secundarios del Pueblo, más cercano a Montoneros. ${ }^{18}$ En muchas de las escuelas de Rosario la UES comenzó a expandirse al fragor de los hechos. Asimismo, hacia mediados de año el Partido Revolucionario de los Trabajadores (PRT) se dio una política de desarrollo en el sector juvenil, del cual surgió la Juventud Guevarista, y que tuvo como objetivo la formación militante de jóvenes que desearan sumarse al partido (Tortolini, 2017, p. 99 y ss.), aunque se desconoce el peso de su inserción en el ámbito escolar en los años posteriores.

El debate por la creación del centro de estudiantes fue un punto de inflexión. En el relato de quienes fueron protagonistas, en general se consideran las primeras reuniones clandestinas pro centro de estudiantes como el inicio de la actividad política en las aulas. Estas reuniones, en la mayoría de los casos, se realizaron 
desde mediados de 1972. Estas iniciativas no estuvieron definidas por una agrupación específica, aunque participaron militantes o cercanos a ellas.

Sobre el Politécnico, Luis señala:

Teníamos quince o dieciséis [años], íbamos a reuniones clandestinas en las aulas de Ingeniería. Había una especie de boxes, íbamos seis o siete y los que aparecían no nos convencían mucho; después descubrimos que eran del PC (...) O sea, me acuerdo que en esa discusión decíamos "Hay que armar el centro de estudiantes" y nosotros decíamos "cómo entre cinco o seis personas vamos a armar el centro de estudiantes”. Entonces ese proceso fue abortado. (...) Ese fue un punto de iniciación en la vida política de uno. ${ }^{19}$

Jorge, que era estudiante del Superior de Comercio, recuerda que para finales del año 1972 había un grupo más o menos organizado vinculado especialmente a la Juventud Peronista, que tenía a su cargo la organización del frente secundario con el objetivo de activar demandas en la escuela y el trabajo frente a las próximas elecciones. ${ }^{20}$ La búsqueda de espacios de participación estudiantil se dio de igual modo en escuelas privadas. Silvia, que iba a la escuela Dante Alighieri, recuerda:

Nos empezamos a acercar sobre todo a un compañero, uno alto grandote que estaba en quinto, que era Horacio, que era del Partido Socialista Auténtico y era uno de los que estaba organizando el centro de estudiantes. (...) Entonces empezábamos a hacer reuniones en... yo no sé... era la Facultad de Medicina (...) y bueno, empezamos a juntarnos. ${ }^{21}$

En algunas escuelas se sumaron los conflictos y problemas internos que eran parte de las primeras agendas y no tenían relación directa con la creación del centro. En la Técnica Provincial $n^{\circ} 1$ un joven fue expulsado acusado del robo de una manzana, hecho que suscitó la movilización por su reincorporación llamada "Manzanazo", ampliamente difundida y relevada. ${ }^{22}$ Estas situaciones, que ya eran un elemento de conflicto, se agudizaron al inicio de la presidencia de Cámpora y es posible pensar que el clima de optimismo que acompañó su asunción dio lugar a la "libre interpretación" del triunfo (Nievas, 2000, p. 60) y que las tomas fueron en esa coyuntura tanto una instancia de apoyo al nuevo gobierno como de reivindicación de las propias demandas. El estudio sobre hechos específicos permite ponderar este aspecto que señalamos, como también aquellos denominadores comunes que atravesaron la experiencia de las ocupaciones, las divergencias y dinámicas propias que cosecharon resultados desiguales.

Si los primeros descontentos y protestas al interior de las instituciones se verificaron hacia fines de abril, como en el caso citado anteriormente, no queda duda de que a partir de junio ya la mayoría de los establecimientos se encontraba bajo control estudiantil. En ese registro, se evidencia que los hechos se precipitaron sin grandes confrontaciones con las autoridades. En Rosario no hay rastros de conflictos tan ostensibles y en general los recuerdos, así como los relatos periodísticos y policiales, tienden a manifestar un cierto grado de acción pacífica, en algunas situaciones con el acuerdo de las autoridades (como en las escuelas Normal 2, Nacional 1 y Dante Alighieri) con las cuales negociaron. En otros casos, con apoyo explícito de preceptores (Escuela Superior de Comercio) y en clara contradicción con las autoridades, especialmente en las instituciones preuniversitarias (Escuela Superior de Comercio e Instituto Politécnico). Sólo se ha identificado en algunas escuelas provinciales el desalojo por parte de autoridades del Ministerio de Educación de la Provincia con el apoyo policial, pero se desconoce si hubo acciones represivas. ${ }^{23}$ Una excepción a ello lo constituye un hecho ocurrido fuera de Rosario, pero que, por su cercanía, tuvo resonancias en esta ciudad. En el intento de hacerse con el control de la escuela Agrotécnica de Casilda, los estudiantes fueron recibidos a balas por el director, quien se encerró en su despacho. La escuela fue tomada con el director encerrado y "la policía ejerciendo discreta vigilancia". ${ }^{24}$

Respecto de la participación política y de las agrupaciones involucradas en ese proceso, vale señalar que tiende a verificarse una pluralidad de voces que matizan y complejizan las lecturas en torno al liderazgo de organizaciones peronistas. Repasemos algunas experiencias para comprender la magnitud que tuvieron. En el 
Nacional 1 la ocupación fue posterior a la creación del centro de estudiantes, espacio donde confluían jóvenes de diversa extracción política:

\footnotetext{
Rápidamente, los que militábamos en política hicimos todos los esfuerzos para tener el centro de estudiantes. Ahí convergimos quienes militábamos en política (...) agrupaciones que ya estábamos constituidos en la escuela, militantes de la JSP, la UES, militantes del PSP [Partido Socialista Popular], militantes de la FJC nos pusimos de acuerdo y armamos una lista que se llamaba Reorganización Nacional, que era una de las consignas del peronismo. Éramos mayoría los peronistas entre los estudiantes. ${ }^{25}$
}

Esta lista se había alzado con el triunfo frente a otra de carácter apartidaria y una de las primeras acciones fue la toma resuelta por asamblea y con acuerdo de la rectora. Es decir que allí no se planteó el cuestionamiento a las autoridades escolares, que más bien acompañaban el reclamo general contra el continuismo. En la escuela privada Dante Alighieri la toma también fue definida por asamblea, en la que participaron estudiantes de diversa extracción política e incluso independientes, y se acordó con el regente. Los objetivos incluían "que se tenga en cuenta el interés y las necesidades del estudiantado. En general lo que se buscaba era un mayor compromiso con la sociedad, o elevar los conocimientos" ${ }^{26} \mathrm{Si}$ bien ambos episodios señalan la convergencia de agrupaciones políticas diversas, es interesante advertir que en la escuela Nacional 1 se había conformado previamente el centro de estudiantes con una lista en la cual confluían diferentes agrupaciones peronistas. Este vínculo sin duda fue efímero, pero advierte sobre las dificultades de cada agrupación para imponerse por su cuenta y la necesidad de construir alianzas frente a un sector estudiantil despolitizado. Asimismo, permite discutir una lectura lineal de los modos de acción de las agrupaciones secundarias peronistas que venían de divergentes trayectorias: la JSP, vinculada más al sector ortodoxo, y la UES, vinculada a la Tendencia Revolucionaria. En esa línea, ya varios autores (Cuchetti, 2010; Besoky, 2013) han señalado la necesidad de dar mayor flexibilidad y discutir la dicotomía derecha/izquierda, con la cual suelen simplificarse las divergencias al interior del peronismo en esa coyuntura.

Diferente es la situación del Instituto Politécnico, escuela dependiente de la universidad, donde el rector designó a Enrique Gomara. Esa intervención iba en consonancia con el recambio institucional que planteaban los nuevos tiempos y era acompañada por el FEN y la JSP. La mayoría del estudiantado, no obstante, se movilizó resistiendo la medida, por considerarla contraria a sus intereses. Eugenio narra:

Y entonces esta cuestión de que salieran en nombre de los estudiantes, poniendo... La intervención del FEN fue muy movilizante y agrandó mucho, e incluyó gente que no tenía nada que ver y que no tenían nada que ver con la izquierda (...), pero había gente de derecha, radicales, gente del PDP [Partido Demócrata Progresista], que nunca habrían participado de esto (...) mientras todos reclamaban la intervención, para que la institución que se tomaba fuera del proceso camporista, nosotros no, nosotros queríamos escuchar a nuestras propias pautas. No era una idea muy redonda, había una idea de que los estudiantes nos definiéramos. Así que tomamos la escuela para oponernos a la intervención. Y esta política de tomar la institución pasaba en todos lados. ${ }^{27}$

Luis, por su parte, recuerda que ante la falta de centro de estudiantes se organizó un cuerpo de delegados que junto a las asambleas definieron la ocupación. Recordemos que el Instituto Politécnico tenía aproximadamente mil quinientos alumnos y los delegados seleccionados (dos por curso) llegaban a más de cien. ${ }^{28}$ Ambos entrevistados destacan que, si bien muchos de los delegados y participantes tenían acercamientos o vínculos con organizaciones políticas, el carácter fue más bien independiente. En ese sentido, debe destacarse que la ocupación de la escuela tenía como un objetivo principal el cuestionamiento a la intervención y no fue liderada por agrupaciones peronistas. En el caso de la UES, porque en esa coyuntura de formación no tuvo una gran cantidad de militantes; la JSP, con un poco más presencia, fue avasallada por el aluvión de estudiantes independientes y de agrupaciones diversas que acompañaron el proceso de la toma y reclamaron consignas que iban a destiempo con los proyectos de intervención que ella acompañaba.

Asimismo, es interesante consignar que cuando los estudiantes se movilizaron hasta el rectorado para protestar contra la intervención de Gomara recibieron el apoyo de compañeros (también fundamentalmente 
varones) de otras escuelas técnicas, lo que señala los vasos comunicantes que permitieron un ir y venir fluido de militancias y debates. Según el memorándum policial, la movilización contó con 150 estudiantes que coreaban "Si no se va Gomara, el industrial no para" y "Acción, acción, afuera la intervención”. ${ }^{29}$ Pese a la masiva movilización en su contra, Gomara asumió como director y se mantuvo en funciones hasta 1977, momento en que fue removido por el rector Riccomi.

En el Superior de Comercio, otra escuela perteneciente a la universidad, se inició la ocupación en la primera quincena de junio, pero se mantuvo al menos hasta finales de julio. El proceso de politización iniciado allí había sido más agudo, y convocó a diversas agrupaciones que emergieron o se visibilizaron al calor de los acontecimientos:

\begin{abstract}
Ahí participaba un montón de organizaciones políticas, de la toma... la UES (...) que se gesta en esos días (...), y había organizaciones... estaba la Federación Juvenil Comunista, estaba... organizaciones trotskistas (...), cada una con, a lo mejor, con una cantidad no muy grande de militantes, pero sí con presencia política (...), había hasta un grupo político, que debe ser uno de los pocos lugares donde tuvo militantes, que era el socialismo revolucionario. Y bueno, ahí tenía dos o tres militantes. Y después estaba la TERS. El Superior era la caldera del diablo (risas), hasta había organizaciones sionistas, organizaciones sionistas de izquierda. ${ }^{30}$
\end{abstract}

La UES, que se había organizado en esos días con pocos militantes, creció significativamente en la institución y lideró el proceso (Di Terlizzi, 2019). Su primer objetivo fue cuestionar la designación del contador Eduardo Barbosa, resuelta desde la gestión de la universidad. La objeción de la UES giraba en torno a que Barbosa "contaba con la recomendación del CGU y del CNU, asesinos de la compañera Silvia Filler en Mar del Plata; de la burocracia sindical y del nacionalismo clerical”. ${ }^{31}$ La prensa señala que en la asunción realizada a fines de junio había estudiantes que "entonaban estribillos partidarios y alusivos a los diferentes sectores del peronismo. Había quienes vivaban por 'la patria socialista' y quienes lo hacían por la 'patria peronista ${ }^{\prime}{ }^{32} \mathrm{El} 5$ de julio se reiniciaron las clases, pero un importante número de estudiantes mantuvieron la protesta y no volvieron a las aulas. Días después el contador Barbosa presentó su renuncia, en coincidencia con el inicio de las vacaciones de invierno. El conflicto se resolvió a finales de mes con la designación del contador Lorenzo Miguel Sambruelli que, sin ser una figura afín a los estudiantes, permitió destrabar el conflicto al acordar con sectores vinculados a la Tendencia Revolucionaria algunos espacios institucionales significativos:

La escuela quedaba un buen porcentaje para la JP: mi caso, el de la vicedirección turno mañana, turno tarde, el departamento contable y de economía, el departamento de lengua (...) y para las 62 organizaciones, el departamento de Matemática y Biología, que estaban juntos (...) y la dirección, que era también para las 62 organizaciones. ${ }^{33}$

Tanto en el Superior de Comercio como en el Politécnico las acciones condujeron a la conformación de los centros de estudiantes en un marco de politización creciente, pero en el Superior también implicaron la redefinición de la gestión, con una instancia de claro avance del peronismo vinculado a la Tendencia Revolucionaria.

Las experiencias recuperadas nos permiten evidenciar la multiplicidad de agrupaciones políticas que convergieron en ese contexto, algunas de vasta trayectoria dentro de las instituciones y otras de creación reciente, pero que fueron centrales en la configuración de ese proceso. Es cierto que las agrupaciones peronistas, y fundamentalmente la UES, capitalizaron el desenlace, pero ello no significó que lideraran las ocupaciones en todas las escuelas o lo hicieran desde el principio. Fundamentalmente, tenemos que reconocer a partir de lo consignado que, si bien la JSP tuvo anclajes significativos en varias instituciones, Nacional 1 y Politécnico, en el primer caso no pudo conducirla y en el segundo se vio desbordada por la movilización. Respecto de la UES, su participación se verificó en muchas escuelas, pero con presencia y mayoría genuina sólo en el Superior de Comercio. Ello se debe a que estaba en instancias germinales de creación dentro del ámbito escolar y terminó por consolidarse a la par o en una etapa posterior. ${ }^{34}$ 
Asimismo, la duración de estas ocupaciones habilita a considerar que la lucha trasvasó la inicial bandera contra el continuismo y mantuvo ciertas consignas que fueron a contrapelo de las posiciones oficiales. El 15 de junio, el secretario General del Movimiento Nacional Justicialista, Juan Manuel Abal Medina, instó a la militancia peronista a desocupar las instalaciones en orden y "controlando que no se produzcan daños contra los bienes del pueblo". ${ }^{35}$ Tres días después un planteo similar sostuvo el ministro del Interior, Esteban Righi; no obstante, las tomas duraron unos días más en la mayoría de las instituciones y en un puñado de ellas se extendieron durante el siguiente mes. Ello permite sostener que las condiciones específicas potenciaron cierta autonomía de las cúpulas dirigenciales, que no veían de buen grado el mantenimiento de estas prácticas a un mes de la asunción de Cámpora y a días del arribo de Perón.

El análisis expuesto, sin ser exhaustivo, evidencia los ritmos diferenciados en los que las instituciones y sus comunidades llegaron a ese acalorado mes de junio y definieron el conflicto. La toma se constituyó en esos breves días en el significante que condensó una pluralidad de demandas estudiantiles, de vínculos y tensiones con las autoridades, de politización estudiantil con sentidos y organización diversos. Aun cuando, como señalamos, el hecho adquirió definiciones particulares en cada escuela, en general implicó la disputa con las autoridades, el cuestionamiento a las normativas institucionales que se cruzaron con transgresiones generacionales en tanto tomar el control de la escuela supuso una afrenta hacia las autoridades y el mundo adulto. Jorge lo define en estos términos:

Lo echamos al director y a los vicedirectores, los echamos a las patadas (...), no dejamos entrar más a los profesores, no dejamos entrar más a los directores, no dejamos entrar a los ordenanzas, no dejamos entrar más a nadie. La escuela quedó en poder de los jóvenes. ${ }^{36}$

En definitiva, habilitó el escenario para resignificar la participación de jóvenes varones y mujeres que cuestionaban el orden en las aulas y fuera de ellas. Los habilitó a pensarse políticamente, con reivindicaciones autónomas, específicas. Como experiencia política, abrió el camino para deliberar sobre cuestiones como la vestimenta, las faltas o la creación del centro de estudiantes. La prensa reseña esa diversidad al señalar que se reclamaban la renuncia de autoridades o de docentes, espacios para biblioteca, laboratorios o "la participación estudiantil en la confección de planes de estudio, el medio boleto estudiantil, el carnet estudiantil, un mayor presupuesto educacional". ${ }^{37}$ Las demandas fundamentales, según las entrevistas, eran tres: eximición con seis, nuevas normativas sobre vestimenta y, en las escuelas universitarias, participar en la elección de la dirección. El cuestionamiento y remoción de autoridades o docentes fue la petición que menos frutos cosechó, ya que, si bien hubo recambio de directivos, no se trató de un correlato de las presiones estudiantiles sino de las modificaciones institucionales en el contexto de un nuevo gobierno.

En muchas escuelas las peticiones menores se lograron, especialmente aquellas que se referían a la modificación de la vestimenta. Esto fue concebido como un logro del movimiento estudiantil y capitalizado por las organizaciones políticas secundarias, y dio un nuevo ritmo a las instituciones educativas y a sus prácticas gestadas entre 1973 y 1974 :

Todo el año 74 habíamos logrado ir sin guardapolvo las mujeres y sin saco y corbata los varones. Eso fue toda una conquista. Porque muchos venían de trabajar, entonces tenían que venir del laburo, sacarse la pintura las mujeres, la... los tacos altos, los varones -muchos trabajaban en talleres, trabajaban...- entonces tenían que venir con el saco, la corbata, ponérselo afuera: O sea, era todo un engorro poder ir a la escuela cumpliendo, digamos... todos estos requisitos. Poder tener el pelo suelto, o sea... son cosas... ir en pantalón, por ejemplo. Un frío de cagarse y las mujeres teníamos que ir en pollera. Íbamos igual porque además éramos de la época de la minifalda; entonces te imaginás, claro: íbamos con las minifaldas, las botas de caña, con los... acordonadas, entonces éramos todas unas vampiresas a la escuela (risas)... viste. ${ }^{38}$

La creación del centro de estudiantes y el desarrollo de elecciones fue otro hecho importante. En el Politécnico, donde había primado una toma con fuerte presencia de sectores independientes y una UES reducida, el centro fue conducido por una agrupación que los entrevistados recuerdan como de "derecha". Recién en 1974 la lista Azul y Blanca conformada por estudiantes de la UES ganó las elecciones. En el 
Nacional 1, donde el centro de estudiantes ya se había constituido, comenzaron a realizarse actividades que incluso excedían la vida institucional. Así, en el año 1973 el centro de estudiantes organizó un recital en una sala provincial: "Lo constituimos con un espacio físico, teníamos nuestra sede (en la sala de celadores), hicimos algunas actividades culturales interesantes, (...) trajimos una obra que era La cantata de La Forestal, como una cuestión social." ${ }^{39}$ En los comicios del Superior de Comercio, la UES demostró su crecimiento: "cuando se constituye el centro de estudiantes, éramos delegados en todos los cursos excepto en dos; mañana, tarde y noche, para que te des una idea de la dimensión que tenía el movimiento peronista y la UES en particular". ${ }^{40}$ Esta preponderancia les permitió "exportar" militantes a otras escuelas con el objetivo de multiplicar experiencias en instituciones políticamente más frágiles.

Otro cambio que trajo aparejada la lucha fue la concreción de espacios de articulación y de encuentro entre estudiantes de las diferentes escuelas. Se promovió la formación de una coordinadora de estudiantes de la ciudad que proponía "llamar a asamblea general de todos los estudiantes para formar definitivamente una organización más amplia y representativa", ${ }^{41}$ la que debía articular una agenda general que involucrara a estudiantes secundarios de la ciudad. La CUES (Coordinadora Única de Estudiantes Secundarios), nombre que adoptó el espacio, se reunió por primera vez en el local del Sindicato de Trabajadores de la Educación de Rosario (SINTER), lo cual evidencia que los primeros vínculos se desarrollaron con el sector combativo de la docencia que había redefinido su identidad como trabajadores y trabajadoras en esta nueva experiencia sindical (Ríos, 2019).

La Coordinadora se convirtió en vaso comunicante, red de información y de relaciones tejidas entre las experiencias de las distintas escuelas a través de asambleas resolutivas en las cuales participaban delegados de cada institución. Además, este espacio se mantuvo vigente en los meses posteriores, asumió reivindicaciones escolares que no habían sido reconocidas e incluso manifestó públicamente su posicionamiento respecto del escenario internacional; por ejemplo, el repudio al golpe de Estado producido en septiembre de 1973 en Chile. Asimismo, llevó adelante distintas actividades públicas entre 1973 y 1975. La más significativa en términos de demanda gremial fue la agitación estudiantil por el medio boleto, bandera central de estudiantes secundarios en esos años. Siguiendo esta línea, es posible pensar que el medio boleto fue una, quizás la más significativa pero no la única, de las consignas gestadas al calor de ese proceso que aunó y movilizó a estudiantes de la amplia geografía nacional.

La activación estudiantil, sin embargo, vivió un período de reflujo muy visible hacia finales del año 1974 y principios del siguiente. La llegada de Oscar Ivanissevich al Ministerio de Educación y las medidas adoptadas marcaron un punto de inflexión en el proceso de politización de los estudiantes secundarios. En enero de 1975, mediante la resolución $n^{\circ} 41$ se suspendió la creación de nuevos centros con el argumento de "evitar una dispersión de esfuerzos o la desnaturalización de los fines a los que deben estar orientados" ${ }^{42}$ Esa fue una de las primeras medidas previas al golpe de Estado de marzo de 1976 que tendieron a modificar la situación en las escuelas; y ya en dictadura, produjo efectos diferenciados de acuerdo con los modos en los cuales cada una transitó este contexto de radicalidad (Luciani, 2017). En esos breves años, el movimiento secundario, si bien no dejó de articular demandas con otros espacios colectivos, especialmente con los universitarios, alentó la coordinación de acciones entre estudiantes de la región y logró visibilizarse como un sujeto con dinámicas propias, e incluso expandirse por escuelas que habían estado menos politizadas en los años previos.

\section{CONCLUSIONES PRELIMINARES}

En este artículo abordamos las ocupaciones de escuelas secundarias producidas en el año 1973. El estudio, a partir de un puñado de experiencias de la ciudad de Rosario, permite discutir algunas lecturas parciales que se han realizado respecto de este tema. En primer lugar, nos permite resignificar el rol que tuvieron los y las jóvenes secundarios en el proceso de movilización que se gestó en los primeros meses del gobierno de 
Cámpora. Fue un acontecimiento que abrió la activación estudiantil a la gestación de demandas en forma autónoma del movimiento universitario. Si bien esta no es la primera vez que presenta una participación activa en el espacio público, es posible pensar que sí es un momento bisagra en la construcción de sus demandas, que articularon algunas que ya tenían su historia e incorporaron una nueva agenda, al mismo tiempo que lograron efectivizar parte de ellas.

En segundo lugar, hemos puesto en evidencia la diversidad de experiencias que convergieron en la ocupación de escuelas. Lejos de asumir que este fue un proceso homogéneo hemos advertido que, junto a esa consigna antidictadura y de lucha contra el continuismo, germinaron dinámicas múltiples que habilitaron la voz y la participación a los y las estudiantes. Asimismo, se evidenció que lo hicieron inscribiendo su militancia en una diversidad de agrupaciones políticas: algunas de extensa trayectoria, otras de creación más reciente. De hecho, muchas de ellas nacieron y todas, incluso las de más extensa vida, crecieron al calor de los sucesos, lo cual nos permite pensar ambos procesos en forma conjunta y paralela. Asimismo, esto deja entrever que, si bien las agrupaciones peronistas, tanto la UES como la JSP, tuvieron una significativa participación y crecimiento, no hegemonizaron el proceso de igual modo ni lo llevaron adelante en todas las escuelas. Si pensamos, en cambio, en el derrotero posterior, efectivamente la UES pudo capitalizar las elecciones de centros de estudiantes en los meses sucesivos.

La ocupación permitió además alentar el lazo entre estudiantes de diversas instituciones, lo que se evidenció en la creación de la Coordinadora. Es cierto que no era la primera vez que se gestaba un espacio de estas características; por el contrario, se asentaba en un proceso acumulativo previo que reponía estas acciones en una trama más general. Los y las estudiantes habilitaron con ello un canal de diálogo con espacios sindicales como el docente al tiempo que expresaban reivindicaciones propias, colectivas, que excedían los problemas en cada colegio. Fue sin dudas un proceso creador de experiencias políticas, radical y democratizador, pero a la vez efímero. La derechización en las instituciones que sobrevino de la mano de la gestión de Oscar Ivanissevich en 1974 y de la dictadura desde marzo del 76 quebró abruptamente ese proceso.

\title{
Prensa y ARCHivos consultados:
}

\author{
Diario El Litoral \\ Diario La Capital \\ Diario La Tribuna \\ El Descamisado
}

Revista Socialismo de Avanzada

Archivo Provincial de la Memoria, Santa Fe

Archivo del Poder Legislativo. Disponible en https://apym.hcdn.gob.ar/expedientes

Ministerio de Educación y Deportes. Repositorio institucional. Disponible en http://repositorio.educac ion.gov.ar/dspace

\section{ENTREVISTAS CITADAS}

Claudia A. Estudiante de la Escuela Normal 2 y participante de la toma. Militante de la FJC entre 1972 y 1976 aproximadamente. Actualmente, es ingeniera agrónoma jubilada, 62 años. La entrevista fue realizada a ella y a una amiga suya, Laura F., en febrero de 2020.

Esteban M. Estudiante del Superior de Comercio a partir de 1971; luego de la toma se trasladó al Instituto comercial Zona Oeste. Militante de la UES. Fue detenido ilegalmente durante junio del 76 y pasó por el centro clandestino de detención conocido como el Servicio de informaciones. Fue luego legalizado y llevado a la cárcel de Coronda como preso político. 64 años. Entrevista realizada en marzo de 2011. 
Eugenio P. Estudió en el Instituto Politécnico entre 1970 y 1974. Estuvo cercano al Movimiento de Acción Secundaria y posteriormente fue miembro de la UES. Fue delegado de curso. Entrevista realizada en abril de 2011.

Gloria C. Estudiante en el Superior de Comercio entre el año 1971 y 1975 . Comenzó a militar en la UES a partir del año 1974. A fines de 1975 fue detenida y quedó a disposición del PEN hasta 1978. Luego salió del país y volvió en la contraofensiva de Montoneros. Regresó definitivamente al país ya en democracia. Entrevista realizada en abril de 2009.

Haydeé S. Docente en el Superior de Comercio luego de la toma realizada en el 73 y hasta 1975, cuando fue cesanteada. Militante de la Juventud Universitaria Peronista (JUP). Entrevista realizada en marzo de 2011.

Jorge P. Estudió en el Superior de Comercio, donde se graduó en 1974. Participó de la toma. Fue delegado de la UES en la escuela incluso hasta después de graduarse. Inició sus estudios universitarios en la Facultad de Ciencias Económicas. Fue detenido ilegalmente en 1976 y posteriormente fue legalizado como preso político. En 1978 se exilió en Venezuela. Actualmente es contador, 60 años. Entrevista realizada en mayo de 2011.

Laura F. Estudiante de la escuela Normal 2, miembro de la UES y posteriormente de la JUP. En la última dictadura fue detenida ilegalmente y luego fue presa política. Actualmente, es docente universitaria, ingeniera y dirigente gremial. 62 años. La entrevista fue realizada a ella y a una amiga suya, Claudia A., en febrero de 2020.

Luis C. Estudiante del Instituto Politécnico entre 1969 y 1973. Participó de las tomas en dicha escuela. Fue luego militante del PST y estudiante universitario. En la década de 1980 fue concejal por el Movimiento Al Socialismo en Rosario. 65 años. Entrevista realizada en agosto de 2011.

Matías L. Estudiante del colegio Nacional 1 entre 1969 y 1973, militante de la Juventud Secundaria Peronista hasta su disolución en 1974 y delegado del centro de estudiantes en el contexto de las tomas. Bancario, 65 años. Entrevista realizada en febrero de 2017.

Silvia C. Realizó parte de sus estudios secundarios en la escuela Dante Alighieri entre 1972 y 1975. Participó de las tomas del colegio. Fue militante del PST, al que se incorporó luego de las tomas. En 1977 retomó la escuela secundaria nocturna en el Nacional 1 y en el año 1978 se radicó en Buenos Aires. Regresó a Rosario ya en democracia. Actualmente, es docente jubilada. 61 años. Entrevista realizada en agosto de 2011.

\section{Material AUdiovisual}

Primer encuentro Talleres de Memorias: 1969: el Rosariazo. Organizado desde CLIHOS y COAD, septiembre de 2019. Disponible en https://www.youtube.com/watch?v=dQGnkwvAFtw

\section{REFERENCIAS}

Águila, G. (2017). El Ejército y el accionar represivo entre dos dictaduras, 1966-1983. En G. Águila (Dir.). (2017). Territorio ocupado. La historia del Comando del II Cuerpo de Ejército en Rosario (1960-1990). Rosario: Editorial de la Municipalidad de Rosario. 99-205

Bartolucci, M. (2008). La primavera del 58. Revueltas, tomas y bataholas juveniles durante el conflicto "Laica o Libre" en Mar del Plata. Recuperado de http://historiapolitica.com/datos/biblioteca/bartolucci2.pdf

Berguier, R., Hecker, E. y Schifrin, A. (1986). Estudiantes secundarios, sociedad y politica. Buenos Aires: Centro Editor de América Latina.

Besoky, J.L. (2013). La derecha peronista en perspectiva. Nuevo Mundo Mundos Nuevos, Questions du temps présent. https://doi.org/10.4000/nuevomundo.65374

Bonavena, P. (2013). El movimiento estudiantil secundario: del Cordobazo a la instalación del Gran Acuerdo Nacional. Jornadas de Sociología de la Facultad de Ciencias Políticas y Sociales Universidad Nacional de Cuyo. Recuperado de https://bdigital.uncu.edu.ar/objetos_digitales/6183/bonavenaponmesa5.pdf 
Bonavena, P. y Millán, M. (2013). ¿ ¿Un movimiento estudiantil moderado en los ’70? El caso de la lucha de los estudiantes de las Escuelas Técnicas contra la "Ley Fantasma" en 1972. Cuadernos del Sur, 41, 37-58. Recuperado de https://revistas.uns.edu.ar/csh/article/view/1625/978

Cammarota, A. (2011). Consideraciones sobre la educación media humanística bajo el primer peronismo (1946-1955): expansión de la matrícula secundaria, inversión estatal y orden educativo meritorio. Temas de bistoria argentina y americana, 19. Recuperado de http://bibliotecadigital.uca.edu.ar/repositorio/revistas/cons ideraciones-educacion-media-humanistica.pdf

Cuchetti, H. (2010). Combatientes de Perón. Herederos de Cristo. Peronismo, religión seculary organizaciones de cuadros. Buenos Aires: Prometeo.

Di Terlizzi, C. (2019). Aulas en movimiento: la historia de la Unión de Estudiantes Secundarios en la escuela Superior de Comercio "Libertador General San Martín" 1973 - 1976. Perspectivas Revista de Ciencias Sociales, 8, 393-414. https://doi.org/10.35305/prcs.v0i8.68

Ferrero, R. (2009). Historia crítica del movimiento estudiantil de Córdoba. Tomo 3 (1955- 1973). Córdoba: Alción Editora.

Gudelevicius, M. (2011). La protesta gremial docente contra el proyecto educativo de la "Revolución Argentina". Archivos de Ciencias de la Educación, 4a época, 5(5). Recuperado de http://www.memoria.fahce.unlp.edu.ar/ar t_revistas/pr.5432/pr.5432.pdf,

Gutiérrez, M. y Pongolini, M. (2015). Participación y activismo de los jóvenes en los centros de estudiantes secundarios en Rafaela, 1972-1976. Una aproximación para el estudio de la historia reciente local. Rafaela: Instituto Superior del Profesorado $\mathrm{N}^{\circ} 2$ "Dr. Joaquín V. González". Mimeo.

Larrondo, M. (2013). Lápices de colores: el movimiento estudiantil secundario en Argentina: investigaciones recientes. Serie Documentos de Trabajo, Red de Posgrados, $n^{\circ}$. 41. Buenos Aires: CLACSO.. Recuperado de http://bibli oteca.clacso.edu.ar/clacso/posgrados/20140120032433/Larrondo.pdf

Lenci, L. (1999). Cámpora al gobierno. Perón al poder. La tendencia revolucionaria del peronismo ante las elecciones del 11 de marzo de 1973. En A. Pucciarelli, La primacia de la politica. Lanusse y la Nueva Izquierda en tiempos del GAN (pp. 167-201). Buenos Aires: Eudeba.

Luciani, L. (2017). Juventud en dictadura. Representaciones, politicas y experiencias juveniles en Rosario (1976-1983). La Plata: UNLP, Facultad de Humanidades y Ciencias de la Educación; Misiones: UNdM; Los Polvorines: UNGS. Recuperado de http://libros.fahce.unlp.edu.ar/index.php/libros/catalog/book/80

Luciani, L. (2018). Las elecciones de 1973 en Argentina: una mirada desde las prácticas sociales. Rúbrica contemporánea, 7(14), 5-19. Recuperado de https://ddd.uab.cat/record/200764

Manzano, V. (2009). Las batallas de los "laicos": movilización estudiantil en Buenos Aires, septiembre-octubre de 1958. Boletin del Instituto de Historia Argentina y Americana "Dr. Emilio Ravignani", n 31, 123-150

Manzano, V. (2011). Cultura, política y movimiento estudiantil secundario en la Argentina de la segunda mitad del siglo XX. Propuesta Educativa, 35, 41-52. Recuperado de https://www.redalyc.org/articulo.oa?id=4030/4030 41706005

Más Rocha, S. M. (2016). El estado y la regulación de la participación estudiantil: la normativa sobre centros de estudiantes secundarios. Polifonias, revista de Educación, año V, n 28, 40-77. Recuperado de http://www.polif oniasrevista.unlu.edu.ar/sites/www.polifoniasrevista.unlu.edu.ar/files/site/Polifonias-8.pdf

Micheletti, M. G. (2018). Laica o libre: las disputas por la creación de las universidades privadas 1955-1959. Rosario: Ediciones Logos.

Nievas, F. (1999). Cámpora: primavera-otoño. Las tomas. En A. Pucciarelli, La primacia de la política. Lanusse y la Nueva Izquierda en tiempos del GAN. Buenos Aires: Eudeba, 351-393.

Nievas, F. (2000). Las tomas durante el gobierno de Cámpora (Tesis de maestría inédita). Buenos Aires: UBA. Mimeo.

Portelli, A. (2003). La orden ya fue ejecutada. Roma, las Fosas Ardeatinas, la memoria. Buenos Aires: Fondo de Cultura Económica.

Pozzi, P. (2012). Esencia y práctica de la Historia Oral. Tempo e Argumento,4(1), 61-70. doi: $10.5965 / 2175180304012012061$. 
Raggio, S. (2017). Memorias de la Noche de los Lápices: Tensiones, variaciones y conflictos en los modos de narrar el pasado reciente. La Plata: UNLP, Facultad de Humanidades y Ciencias de la Educación; Posadas: UNdM; Los Polvorines: UNGS. Recuperado de http://libros.fahce.unlp.edu.ar/index.php/libros/catalog/book/98

Ríos, G. (2019). Hora de abrir los ojos. El proceso de sindicalización docente en la provincia de Santa Fe, 1969-1976. Rosario: Homo Sapiens.

Rodríguez, L. (2019). Cien años de normalismo en Argentina (1870-1970). Apuntes sobre una burocracia destinada a la formación de docentes. Ciencia, Docencia, Tecnologia, 30, no 59 nov-ab,. 200-235 https://doi.org/10.3325 $5 / 3059 / 690$

Sanguinetti, A. (2013). El devotazo. Buenos Aires: Colección Documentos-Colectivo El Topo Blindado. chttp:// www.peronlibros.com.ar/editoriales/1160/coleccion_documentos-colectivo_el_topo_blindado

Scocco, M. (2018). Comisiones investigadoras en las transiciones democráticas en Argentina. El caso de la provincia de Santa Fe. Rubrica Contemporanea, 7(14), 21-42. Disponible en .

Seoane, M. y Ruiz Núñez, H. (1992). La noche de los lápices. Buenos Aires: Planeta.

Svampa, M. (2003). El populismo imposible y sus actores. En D. James, Nueva Historia Argentina, 1955-1976. Volumen IX. Buenos Aires: Sudamericana, 386-436.

Thompson, P. (2005). Historia Oral y Contemporaneidad. En Anuario no 20, Rosario: Homo Sapiens.

Tortolini, V. (2017). Política de masas para una estrategia revolucionaria: PRT-ERP, 1973-1976. Avances del Cesor, XIV, 16, 91-110. Recuperado de http://web2.rosario-conicet.gov.ar/ojs/index.php/AvancesCesor/index

\section{Notas}

1 De carácter general, destacamos dos estudios: una obra ya clásica (Berguier, Hecker y Schifrin 1986) y un trabajo sobre el movimiento secundario en la segunda mitad del siglo XX (Manzano, 2011). Asimismo, hay investigaciones parciales sobre coyunturas específicas que retomaremos a lo largo del artículo. No obstante, es interesante consignar que, sin abordar específicamente sus experiencias de movilización, las investigaciones más difundidas sobre estudiantes secundarios se refieren a la represión durante la última dictadura y a las memorias posteriores (Seoane y Núñez, 1992; Raggio, 2017).

2 Los aportes de la historia oral han sido señalados por referentes en el área (Portelli, 2003; Thompson, 2005; Pozzi, 2012). En esta investigación, las entrevistas permitieron recabar información y, fundamentalmente, recuperar recuerdos de los y las entrevistados/as. Hasta el momento se ha entrevistado a 14 personas. 10 de ellas fueron participantes de la ocupación ( 6 varones y 4 mujeres) que tenían militancia previa o la asumieron en ese contexto; 3 entrevistas se realizaron a quienes no tenían ni tuvieron militancia política: una de ellas, una mujer, no participó directamente de la toma, aunque la apoyaba; de los dos varones, uno fue participante y el otro no. También se entrevistó a una docente que asumió funciones a partir de 1973 y fue cesanteada en 1975. Para el desarrollo general del trabajo, se utilizaron otras entrevistas realizadas para proyectos anteriores, especialmente a aquellas personas que ingresaron a la escuela secundaria entre 1975 y 1977.

3 Las escuelas a las cuales hacemos referencias eran de larga trayectoria en la ciudad de Rosario. Las escuelas normales 1 y 2 eran de mujeres, mientras que la 3 y el nacional 1, la primera escuela de la ciudad, [Supongo que sería "por entonces sólo era"] de varones.

4 CLIHOS-UNR/COAD (2019, 11 de septiembre). Talleres de memoria. 1969: el Rosariazo. Primer encuentro. Rosario. [Archivo de video] Recuperado de https://www.youtube.com/watch?v=dQGnkwvAFtw

5 Claudia C.

6 El Descamisado, año I, $\mathrm{n}^{\circ}$ 3, junio de 1973.

7 Cabe recordar que, a nivel nacional, las tomas de instituciones públicas y privadas se iniciaron en el mes de mayo luego de la asunción de Cámpora a la presidencia. Algunas cifras muestran la magnitud de esta forma de protesta: en el breve lapso de dos meses, entre finales de mayo y julio, se registraron al menos 2000 ocupaciones.

8 Balestra y otros (1973). Pedidos de informes a los Ministros del Interior, Justicia y Cultura y Educación sobre las tomas y apropiaciones de establecimientos y bienes públicos y privados. Expediente $\mathrm{n}^{\circ}$ 435. Archivo del Poder Legislativo. Recuperado de https://apym.hcdn.gob.ar/expedientes

9 El Litoral, 12 de junio de 1973.

10 Entrevistas e informes del APM permiten señalar que hubo ocupaciones en distintas ciudades de la región, desde aquellas cercanas a las grandes urbes (por ejemplo, las del cordón industrial de Rosario) pero también de otros departamentos 
del sur y el norte de la provincia. También algunos trabajos señalan que, sin llegar a la toma, algunas escuelas vivieron en ese período un proceso de activación estudiantil (Gutiérrez y Pongolini, 2015).

11 Memorándum (1973, 14 de julio). Unidad de Conservación (UC) 41, folio 1. Archivo Provincial de la Memoria (APM). La noticia sobre las escuelas tomadas también se difundió en los medios locales en la misma fecha. Fueron ocupadas las escuelas Madre Cabrini, Cristo Rey, Medalla Milagrosa y San José.

12 El Litoral, 12 de diciembre de 1972.

13 Asunto: Ocupaciones. UC82, f.2. APM. La información es consignada por la DGI mediante el recorte de prensa.

14 Memorándum n 2252, 11 de abril de 1973, UC, 53A, f. 2. APM.

15 Memorándum s/n, 12 de abril de 1973, UC 53B, fs. 1 y 2. APM.

16 El Litoral, 12 de abril de 1973, p. 5.

17 En este apartado se referencia la ocupación de algunas escuelas de la ciudad para analizar la diversidad de experiencias. Por ello analizamos la escuela Nacional 1, escuela ya tradicional de la ciudad; Politécnico y Superior de Comercio, dependientes de la Universidad; la escuela Normal n ${ }^{\circ} 2$ y una escuela privada (Dante Alighieri). Asimismo, se hará referencia a escuelas técnicas dependientes de CONET y provinciales.

18 Laura F.

19 Luis C.

20 Jorge $P$.

21 Silvia C. Probablemente se refiera a un militante del Partido Socialista Popular.

22 Juventud de Avanzada Socialista, n 62, junio de 1973. También Memorándum n² 2268, 4 de mayo de 1973, UC 53A, f.2. APM.

23 Parte de informaciones, 26 de junio de 1973, UC 411, APM.

24 La Tribuna, 13 de junio de 1973, tapa.

25 Matías L.

26 Silvia C. Recuerda la participación de estudiantes militantes peronistas y de agrupaciones de izquierda. Ella aún no militaba en ningún espacio político.

27 Eugenio P.

28 Luis C.

29 Memorándum s/n, 25 de junio de 1973. UC 53B, f 1. APM.

30 Jorge $P$.

31 Memorándum s/n, 6 de julio de 1973, UC 41, f.1. APM.

32 La Capital, 30 de junio de 73 p. 6.

33 Haydeé $S$.

34 Aun cuando en este artículo sostenemos que la ocupación fue un hito significativo en la lucha del movimiento estudiantil secundario, retomamos algo ya señalado: la participación no involucró al total de estudiantes y muchos, si bien se vieron afectados, no así interpelados. Laura recuerda que el Normal 1 fue tomado por estudiantes del Profesorado que funcionaba en las mismas instalaciones, por lo cual no generó la activación entre sus compañeras secundarias. No obstante, ella y otras pocas militantes participaron en otras instituciones

35 El Litoral, 15 de junio de 1973.

36 Jorge $\mathrm{P}$.

37 La Tribuna, 12 de junio de 1973.

38 Gloria C.

39 Matías L.

40 Esteban M.

41 Esteba M.

42 Resolución n 41, enero de 1975. Ministerio de Cultura y Educación. Recuperado de http://repositorio.educacion.go v.ar/dspace/handle/123456789/88853 